\title{
eJRIEPS
}

Ejournal de la recherche sur l'intervention en éducation physique et sport

$23 \mid 2011$

Varia

\section{Les registres de technicité : un concept utile pour analyser l'activité des sujets dans les APSA ?}

\section{Alain Mouchet}

\section{OpenEdition}

\section{Journals}

Édition électronique

URL : http://journals.openedition.org/ejrieps/4550

DOI : 10.4000/ejrieps.4550

ISSN : 2105-0821

Éditeur

ELLIADD

\section{Référence électronique}

Alain Mouchet, «Les registres de technicité : un concept utile pour analyser l'activité des sujets dans les APSA ?», eJRIEPS [En ligne], 23 | 2011, mis en ligne le 01 avril 2011, consulté le 21 février 2020. URL : http://journals.openedition.org/ejrieps/4550 ; DOI : 10.4000/ejrieps.4550

\section{(c) (1)}

La revue eJRIEPS est mise à disposition selon les termes de la Creative Commons Attribution 4.0 International License. 


\section{Les registres de technicité :}

un concept utile pour analyser l'activité des sujets dans les APSA?

Alain Mouchet

Université Paris Est Créteil Val de Marne, Département STAPS, CIRCEFT (EA 4384), équipe REV, France

\section{Résumé}

Le but de cet article est d'examiner l'usage du concept de registre de technicité comme grille d'analyse de l'activité des sujets dans les APSA. Nous revenons sur l'évolution des définitions de la technicité et des registres de technicité, importés dans le champ des STAPS, afin de repérer les différentes interprétations et les éléments stables. Ensuite l'enjeu est d'illustrer le caractère fonctionnel de ce concept dans le champ sportif à travers l'analyse de l'activité d'entraîneurs en match et de l'activité décisionnelle de joueurs. Nous mettons en avant la possibilité de spécifier les différents registres, ainsi que l'articulation entre ces derniers. II nous paraît intéressant de continuer à approfondir l'exploitation de ce concept outil en recherche et en formation, dans des contextes variés liés notamment à I'APSA et aux caractéristiques des situations analysées.

\section{Introduction}

En 2010, Mouchet, Amans-Passaga et Gréhaigne présentent un état des lieux sur l'approche technologique à travers les fondements épistémologiques et théoriques, les caractéristiques méthodologiques, ainsi que des résultats de travaux réalisés dans différents secteurs d'intervention en Activités Physiques Sportives et Artistiques (APSA). Mais les textes fondateurs de l'approche technologique en STAPS sont repérables au début des années quatre-vingt dix à travers les propositions de Bouthier (1993), Bouthier, Durey (1994), Gréhaigne, Bouthier (1994), qui impulsent cette orientation de recherche. Bouthier (1993) considère que les recherches technologiques sont des études sur les conditions de transmission et/ou d'appropriation des techniques corporelles, dans leur système sociotechnique d'apparition. Ces recherches servent les objectifs suivants: accroître les connaissances sur l'intervention, contribuer à la constitution de savoirs sur la formalisation et la transformation des techniques corporelles, contribuer à l'optimisation des procédures d'entraînement, de formation et d'enseignement. 


\section{eJRIEPS 23 avril 2011}

C'est dans le cadre de ces objectifs que nous proposons de revisiter le concept de registre de technicité. Force est de constater que ce dernier a été peu mobilisé dans les recherches en STAPS excepté dans les travaux de Gérard, Bouthier (2004) ou Uhlrich, Bouthier (2008) à propos du registre de lecture exploité dans l'observation des conduites motrices de pratiquants. Mais à notre connaissance ces études ont peu précisé l'idée initiale d'interaction entre les quatre registres de technicité tels que définis par Bouthier, Durey (1994).

Le but de cet article est alors de discuter le caractère fonctionnel de ce concept pour analyser l'activité technique de sujets engagés dans une APSA. Nous ferons tout d'abord un examen des travaux originels qui ont amené l'apparition de cette notion dans le champ des STAPS, afin d'étudier sa dynamique évolutive. Puis nous proposerons des exemples d'exploitation de cet outil d'analyse dans le secteur sportif en formalisant les compétences d'entraîneurs en match, ou encore les conduites décisionnelles de joueurs de rugby. Nous ferons alors une relecture de résultats obtenus dans le cadre de travaux antérieurs. Cette nouvelle interprétation des données vise à mieux mettre en lumière l'organisation de l'activité des sujets dans ses dimensions complexes et dynamiques.

\section{Ancrages conceptuels}

1. 1. Subjectivité dans l'activité technique

Dans le but de décrire et/ou comprendre l'activité technique déployée par les sujets dans leur contexte de pratique, plusieurs auteurs mettent en avant une conception riche et rénovée de la technique (Heidegger, 1958 ; Amade-Escot, Bos, Dufor, Dugrand, Orphee, Terrisse, 1994 ; Durey, 1995 ; Bouthier, 1995). II s'agit d'une technique incorporée ou incarnée, indissociable d'une pratique singulière et qui relève plutôt de la Technê. C'est à dire d'une création artisanale liée à un usage particulier relative à une fin qui est intégrée dans un sens global. Les gestes relèvent d'une connaissance pratique, ils dévoilent l'usage et l'ouvrage d'un auteur/acteur, c'est-à-dire qu'ils caractérisent le sujet de la technique.

Ainsi, en plus de la rationalité technique formalisée, canonique et impersonnelle (par exemple les techniques de plaquage), existent des pratiques singulières, individualisées, disposant d'une propre cohérence organisatrice des conduites motrices. Notons que cette facette subjective est sans doute sous-jacente dans la distinction et la dynamique établies par Leplat, Pailhous (1981) entre habileté et technique. De même, Bouthier (1993) a élaboré une modélisation des composantes de l'action sportive d'un sujet, qui relève de cette conception rénovée des techniques. Cette formalisation est très fonctionnelle pour 


\section{eJRIEPS 23 avril 2011}

analyser l'activité de sujets engagés dans une activité physique, que ce soit des pratiquants ou des intervenants.

Plus récemment, l'évolution des préoccupations de recherche, des cadres scientifiques et des méthodes d'analyse, place clairement la subjectivité au cœur de certaines études sur l'activité des joueurs en sports collectifs. Mouchet (2008a) à propos des joueurs de rugby, pointe la facette subjective dans l'organisation des prises d'information et des décisions. II souligne d'une part, les rapports subjectifs entretenus par les joueurs avec les éléments communs au groupe (projet de jeu et projet d'équipe, référentiel commun). D'autre part, une logique personnelle en cours d'action, qui peut être différente d'une logique théorique ou rationnelle du jeu. Eloi (2009) souligne l'existence chez des passeurs de haut niveau en volley-ball, de styles décisionnels qui valorisent des combinaisons personnalisées entre les pôles stratégiques et tactiques d'une part, technique d'autre part.

Ces travaux mettent en avant les composantes fines et tacites des compétences en acte ; ils renseignent la dimension privée de l'activité technique des sujets, de manière complémentaire à la dimension publique des actions qui peut être objectivée par des outils vidéo informatiques.

Par conséquent, nous considérons que l'activité technique engage l'individu dans toutes ses dimensions, et les productions techniques des pratiquants relèvent d'une construction personnelle élaborée. L'enjeu pour un intervenant ou pour un chercheur, est de disposer de moyens d'analyse de l'activité de l'individu dans sa complexité, sa subjectivité, sa singularité. Les registres de technicité peuvent-ils répondre à cet enjeu ?

\subsection{Technicité}

Pour Combarnous (1982), les activités techniques reposent sur l'existence d'une aptitude de l'homme à résoudre des problèmes concrets d'une manière originale : les hommes ont constitué des associations de connaissances raisonnées et empiriques, toutes éprouvées par la pratique, qui assurent l'efficacité des actions et permettent de concevoir, de réaliser, d'utiliser des dispositifs de caractère technique. Ce caractère technique est la technicité. Ce concept offre des perspectives originales pour l'analyse de l'activité technique. «Dans le langage courant le mot technicité englobe des aptitudes aux créations techniques, des comportements favorables à leurs emplois et des capacités de compréhension des réalisations. La technicité est donc à la fois une aptitude individuelle et un comportement collectif. ${ }^{1}$ Combarnous (1982) identifie ainsi trois composantes dans la technicité :

\footnotetext{
${ }^{1}$ Combarnous, M., La culture face à la technique, pp.227-228.
} 


\section{eJRIEPS 23 avril 2011}

- une pensée propre, la rationalité technique. C'est une forme de réflexion et de construction d'idées incluant des raisonnements. La coexistence du savoir-faire, de l'utilisation de recettes et de l'adoption des résultats scientifiques caractérise cette rationalité technique ;

- un composant matériel, l'emploi d'engins (outils, instruments, machines, équipements), comme intermédiaire obligatoire entre une pensée et une action ;

- une spécialisation des tâches et une organisation de l'activité de production. Les diffusions et les propagations des techniques font apparaître l'étroite dépendance qui lie les techniques aux cadres matériels, humains et sociaux dans lesquels elles se trouvent.

Pour Combarnous (1982), la réunion de ces composantes est un système qui constitue la base de toutes les activités techniques. Afin d'illustrer ce système dans le champ sportif, nous pouvons dire qu'en ce qui concerne la rationalité technique, Mouchet, Uhlrich, Bouthier (2008) identifient une relative diversité des modèles de référence, pragmatiques et théoriques, à propos du jeu de mouvement en rugby. La composante matérielle est mise en avant dans les travaux d'Uhlrich (2005) à propos du Pocket Assistant Coach en rugby. C'est un outil d'analyse du jeu de mouvement et d'aide à la décision pour les entraîneurs. Cet outil mobilise la rationalité technique pour établir des catégories d'analyse du jeu produit et contribue en retour à une évolution des modes de pensée sur le jeu. Enfin, pour caractériser la dernière composante, le PAC rugby nécessite l'apprentissage de rôles sociaux avec des binômes d'observateur, et son développement en tant que produit exige la mise au point d'un réseau de collaborateurs dans différents secteurs sociaux de pratique (Fédération Française de Rugby, monde de l'EPS...).

Martinand (1994) reprend le concept de technicité dans le cadre de l'enseignement et plus spécialement à propos des disciplines technologiques. II propose un modèle de formation des enseignants, « plus propre à la formulation des problèmes de conception curriculaire et de formation des enseignants $»^{2}$. Deux ambitions guident cette proposition :

- la spécificité, la complexité de l'activité de l'enseignant, à la base de sa "professionnalité" doivent pouvoir être prises en compte et analysées de façon plus profonde ;

- le rapport de l'école aux domaines de référence non scolaires, où les acquis scolaires doivent pouvoir prendre sens, doit être élucidé de façon plus radicale.

\footnotetext{
${ }^{2}$ Martinand, JL., La didactique des sciences et de la technologie et la formation des enseignants, p.67.
} 


\section{eJRIEPS 23 avril 2011}

Ces deux ambitions conduisent à deux distinctions, d'une part entre pratiques et disciplines, d'autre part entre école et référence. Aussi, pour caractériser ces pratiques, il ne suffît pas de prendre en considération les savoirs en jeu : objets et instruments, tâches et problèmes, qualifications et rôles sociaux constituent avec les savoirs, les composantes solidaires d'une pratique. Au cœur des pratiques de référence, sont les technicités. Elles participent aux débats éducatifs et aux décisions curriculaires : écarts entre technicité dans les références choisies et les contenus des disciplines scolaires, rapports entre pratiques familières aux apprenants et pratiques de référence, sens des activités et des apprentissages. Ainsi selon Martinand (1994), les enseignants devraient acquérir une double technicité, dans les pratiques de référence d'une part et dans le guidage des activités et des apprentissages scolaires d'autre part.

\section{3. Registre de technicité}

Martinand (1994) suggère une évolution conceptuelle en développant l'idée de registres de technicité. Les registres de technicité caractérisent et définissent l'action d'un enseignant, ses compétences techniques exercées dans les pratiques scolaires, en relation avec ceux des pratiques sociotechniques de référence. Glomeron (2001) s'inscrit dans cette perspective et estime que la technicité constitue un élément de la culture commune qui doit être partagée par les enseignants. Les pratiques enseignantes sont ainsi fortement référencées à la discipline scolaire et aux pratiques sociotechniques. C'est par rapport à ces dernières que se pose le problème des registres de technicité, puisque ce rapport ne peut pas être uniquement pensé en termes de maîtrise de l'ensemble de ces pratiques. En effet, le champ des possibles est beaucoup trop important pour en assurer la maîtrise. Quatre registres de technicité sont distingués par Martinand (1994) :

- registre de la maîtrise : c'est le seul pris habituellement en compte, dans les pédagogies de la maîtrise. Glomeron (2001) précisera que c'est celui du professionnel qui intervient en autonomie dans une pratique ;

- registre de la participation : il apparaît lorsqu'il y a capacité à tenir un rôle dans une pratique, mais avec aide et contrôle par autrui;

- registre de l'interprétation : c'est celui qui fonctionne lorsque sans être forcément capable de faire soi-même. On a la capacité de "lire" et d'analyser, d'expliquer une pratique ;

- registre de la modification : c'est celui qui permet de transformer une pratique, ce qui suppose que la maîtrise de cette dernière soit partielle et non consolidée. 


\section{eJRIEPS 23 avril 2011}

II s'agit bien de registres, et non de niveaux, car il n'y a pas hiérarchie sur une dimension. Chaque registre comporte des compétences, des représentations et des valeurs spécifiques.

Dans le secteur de l'EPS et en STAPS, Bouthier (1993) répertorie et définit ainsi :

- le registre de la maîtrise des techniques sportives existantes dans la spécialité donnée. II recouvre la possibilité des pratiquants expérimentés d'être performant par l'appropriation très affinée des techniques corporelles connues ;

- le registre de participation qui suppose en tant qu'acteur de pouvoir tenir différents rôles ou fonctions dans l'activité sportive. Notons que Bouthier, Durey (1994) précisent que la contribution de certains pratiquants en sports collectifs, qui font nombre en intervenant très rarement sur la balle et l'adversaire, peut renvoyer à ce registre. Mais aussi toutes les situations aménagées où il s'agit simplement de réaliser une partie du processus, l'ensemble étant maîtrisé par d'autres ;

- le registre de transformation des techniques sportives en usage par des créations nouvelles qui dépassent la simple reproduction des modèles établis, mais qui se font en référence aux modèles antécédents. Les techniques sont par essence des solutions pratiques imaginées par les hommes. Signalons que Bouthier, Durey (1994) ajoutent l'idée que lorsqu'il s'agit d'agir sur les techniques mises en œuvre par un tiers c'est précisément le domaine de l'intervention.

- le registre de lecture des productions sportives qui correspond à la capacité d'identifier les différentes composantes caractéristiques de la pratique, et d'utiliser pour les analyser les concepts et outils technologiques spécifiques comme peuvent le faire les pratiquants mais aussi les entraîneurs ou les spectateurs avertis.

Ce sont ces définitions qu'Uhlrich (2005) mobilise dans sa thèse sur le rôle des artefacts matériels et cognitifs dans le développement des compétences de l'intervenant éducatif en rugby. Uhrich, Bouthier (2008) ajouteront à propos du registre de lecture qu'il suppose la possibilité de mobiliser des outils matériels comme des fiches d'observation et mentaux élaborés par des spécialistes du domaine.

Par conséquent nous pouvons repérer une dynamique dans l'évolution relative de ce concept de registre de technicité :

- le registre de maîtrise correspond à la mise en œuvre efficace et autonome des techniques. La définition reste stable dans les différentes propositions des auteurs ;

- Le registre de participation peut correspondre à l'activité déployée par un sujet dans une temporalité immédiate (situation, leçon, match...), ou dans une temporalité plus longue (cycle d'enseignement ou de formation, année scolaire ou saison sportive) ; 


\section{eJRIEPS 23 avril 2011}

- Le registre de « modification » (Martinand, 1994) évolue vers la «transformation » (Bouthier, Durey, 1994) des techniques. Ce changement terminologique traduit selon nous une double interprétation possible dans la nature des transformations et des temporalités différentes. Nous pensons à un ajustement en cours de situation, ou à une évolution plus lourde, s'inscrivant dans le long terme comme c'est le cas dans l'invention de techniques nouvelles;

- Le registre de «l'interprétation » évolue vers la « lecture ». Nous considérons que le terme d'interprétation était plus englobant et intégrait la seule lecture de la motricité. Cette notion initiale nous semble plus révélatrice de la dimension complexe et subjective de l'activité technique, telle que nous l'avons soulignée précédemment. En effet cela recouvre la construction personnelle dans l'observation et l'analyse d'une situation ou de la motricité des sujets. Nous pourrions sinon remplacer lecture par observation et analyse.

\section{4. Synthèse sur l'évolution des concepts}

Nous pouvons donc repérer que la signification et l'utilisation des concepts évoluent selon le contexte d'utilisation. Ainsi la technicité chez Martinand (1994) est réduite à l'activité des enseignants avec des préoccupations curriculaires et de formation. II s'agit d'une double technicité, dans les pratiques sociales de référence et de guidage. Alors que le sens initial chez Combarnous (1982) concerne d'autres publics et contextes; il correspond de manière plus générale au caractère technique de toute activité humaine, avec une dimension rationnelle, instrumentale et sociale.

Le concept de registres de technicité, initialement proposé par Martinand (1994) pour la didactique de l'enseignement technologique et des APSA, évolue lui aussi lors de son usage à propos des pratiques physiques. Bouthier, Durey (1994) évoquent toujours l'enseignant, mais aussi de manière plus large l'intervenant (y compris l'entraîneur) et le pratiquant, élève ou sportif, donc d'autres sujets. Les registres concernent alors l'acte d'intervention dans sa dialectique intervenant/apprenants, ainsi que les perspectives de formation des intervenants, rejoignant ainsi la pensée originelle de Martinand (1994). Nous pouvons penser que les échanges entre ces auteurs furent favorisés par leur collaboration au sein d'une option de DEA et de la formation doctorale en Didactique des Activités Physiques et Sportives (ENS Cachan, Université Paris 11, INRP). Notons également l'éclairage complémentaire et utile des travaux de Glomeron (2001) sur les critères d'identification des registres de technicité. II existe un registre lorsque une technicité réelle est mise en œuvre dans l'action ; qu'il est exprimé par rapport à une pratique 


\section{eJRIEPS 23 avril 2011}

sociotechnique prise en référence ; qu'il est généralisable à un ensemble de situations et d'acteurs ; qu'il correspond à un processus d'action cohérent et reproductible pour l'acteur. Nous estimons par conséquent que l'extension de l'usage de ce concept au-delà de la formation des enseignants des disciplines technologiques est pertinente et fonctionnelle dans le champ des APSA. L'acception que nous proposons concerne donc la dimension publique et privée de l'activité technique des sujets, intervenants et apprenants, dans leurs relations à des pratiques sociales de référence. Le versant relatif à l'intervenant concerne bien l'idée initiale de sa professionnalité qui s'exerce dans différents registres de technicité. C'est une activité sociale (diffusion, transmission, appropriation, utilisation, création...), avec l'existence de techniques partagées, formalisées, évolutives, inhérentes aux APSA. Mais c'est aussi une activité technique où nous pointons la dimension personnelle, subjective, au sens psychophénoménologique (Vermersch, 2003) de la logique propre du sujet.

Nous suggérons alors d'utiliser le concept de registre de technicité pour caractériser et rendre intelligible l'activité technique des apprenants, pour mettre en évidence du point de vue d'un chercheur mais aussi d'un intervenant, le fonctionnement propre du sujet dans son rapport à la culture technique. Rappelons d'ailleurs que chez Combarnous (1982), le terme de culture présente deux significations extrêmes, l'une sociale et l'autre individuelle. La signification collective correspond à ce qui, pour un groupe ou une société constitue un bien commun, est admis par l'ensemble, sert d'appui ou de guide aussi bien aux réactions globales qu'à un très grand nombre de réactions personnelles. Dans le domaine de la culture technique, les techniques n'ont d'existence que par leurs ancrages dans les groupements humains, ancrages qui assurent en particulier, la continuité entre les générations. Les diffusions et les propagations des techniques, qui se sont effectuées constamment au cours des siècles, font apparaître l'étroite dépendance qui lie les techniques aux cadres matériels, humains et sociaux dans lesquels elles se trouvent. La signification individuelle correspond pour chaque individu à ce qui est assimilé et imprègne son esprit; cette culture est différente d'un individu à un autre ; et pour une même personne elle varie dans le temps ; elle s'enrichit par l'éducation, par l'expérience, par les réflexions personnelles, en un mot par «la vie». Ainsi donc les cultures sont évolutives parce que composées d'éléments eux-mêmes en incessante évolution.

\section{Illustrations dans le secteur sportif}

2. 1. Activité des entraîneurs en match 


\section{eJRIEPS 23 avril 2011}

«Le domaine de l'intervention pour transmettre et faire reconstruire des techniques corporelles constitue un autre ensemble de registres de technicité spécifique à affiner. La connaissance des registres de technicité est à la fois un enjeu scientifique en soi et une nécessité dans le cadre de la problématique didactique de transmission-appropriation des connaissances techniques sportives. ${ }^{3}$

Nous nous inscrivons dans cette perspective à travers une étude effectuée en réponse à une commande de la Cellule Recherche Rugby de la DTN de la Fédération Française de Rugby. Nous avons étudié les compétences des entraîneurs de sélections nationales en ce qui concerne le coaching, entendu comme l'activité en match (Mouchet, 2008b). L'échantillon concernait la sélection de France Amateurs lors d'un match contre l'Angleterre, l'équipe des moins de 21 ans à l'occasion du Championnat du Monde 2006, France $A$ féminines contre les Pays-Bas, et France féminine à 7 lors du Championnat d'Europe 2008.

Nous constatons par exemple à propos du registre de maîtrise des techniques d'intervention, que les entraîneurs déploient des routines de fonctionnement qui leur paraissent efficaces. C'est le cas lors de la gestion des changements de joueurs en appliquant des stratégies préétablies qui ont été efficaces dans des compétitions antérieures. Mais aussi en respectant les remplacements prévus à l'avance, ou en anticipant et en écrivant un scénario de blessures pour ne pas avoir à trop subir de désagréments. Ils préservent donc un fonctionnement qui tend à réduire l'incertitude. C'est également le cas lors de leurs interventions auprès des joueurs en match (Mouchet, Le Guellec, 2010). Sur ce dernier point nous repérons lors d'études de cas avec les deux entraîneurs d'une même équipe, des stratégies préférentielles de fonctionnement. Ces modes de fonctionnement sont mis en œuvre dans des matchs dont le contexte est pourtant différent (rapport de force, enjeu du match, placement par rapport au terrain...). Ainsi un entraîneur est caractérisé par la volonté d'influer sur la rencontre par un mode d'intervention directif avec :

- Un volume très important de communications, principalement en cours de jeu ;

- L'utilisation prédominante des cris et interpellations ;

- Une centration prioritaire sur les aspects stratégiques et tactiques ;

- Un fonctionnement réactif, autonome et efficace deux fois sur trois ;

- Des intentions prioritaires consistant à imposer et guider l'exécution des joueurs.

\footnotetext{
${ }^{3}$ Bouthier, D., L'approche technologique en STAPS, représentations et actions en didactique des APS, p.100.
} 


\section{eJRIEPS 23 avril 2011}

Mais au-delà de ce registre de maîtrise, nous pointons aussi dans les différents staffs techniques, le registre de transformation des techniques d'intervention à travers les éléments suivants :

- Des tentatives de gestion innovante des remplacements (entrée programmée et entérinée de toutes les remplaçantes à la mi-temps dans un contexte a priori peu risqué, adaptation du scénario envisagé à la suite d'une blessure imprévue, entrée précoce et inhabituelle de nombreux joueurs en même temps) ;

- L'organisation et le contenu inhabituels des discours d'avant match ou à la mitemps (fibre émotionnelle plus fortement sollicitée qu'à l'accoutumée) ;

- La transmission des consignes via des intermédiaires (kiné, porteur d'eau, joueurs relais) ou s'appuyant sur des joueurs leaders responsabilisés par secteur de jeu.

Par ailleurs, le registre de lecture est fortement sollicité lors de l'observation du jeu en début de match (Mouchet, 2010). Le fonctionnement par phénomène d'œil de maquignon, repose en fait sur des cadrages attentionnels organisés qui articulent des repères d'arrièreplan et des indices significatifs en situation. Par exemple un entraîneur ${ }^{4}$ évalue la partie au regard de ses prévisions en mobilisant les indicateurs suivants :

- La combinaison d'indices observés en situation, de communications avec le manager dans les tribunes via la radio, et de données d'arrière-plan décisionnel à propos des caractéristiques des joueurs et du fonctionnement de l'équipe ;

- L'absence de répétition d'erreurs, ce qui constitue pour lui un indicateur important au regard du fonctionnement de l'équipe ;

- Des éléments du contexte local comme la météo (vent favorable en deuxième mitemps) et la stratégie de remplacement des joueurs sur ce match (entrée de bons joueurs prévue en deuxième mi-temps).

De plus, il effectue une analyse systémique du rapport d'opposition à travers les points forts et points faibles des deux équipes, à travers la globalité et certains détails du jeu, et en observant les différentes phases de jeu (conquêtes, fixations, mouvement). Cette analyse lui permet de dresser un bilan favorable aux Français, même si le score serré, et de prendre la décision de ne pas intervenir auprès des joueurs. Cet entraîneur privilégie la pensée logique, rationnelle, délibératoire.

\footnotetext{
${ }^{4}$ Il s'agit d'un entraîneur de l'équipe de France des moins de 21 ans, à l'occasion d'un match contre le Pays de Galles lors du Championnat du monde 2006 qui s'est déroulé en France. La France mène 6/3 à la mitemps, puis gagne le match $32 / 3$.
} 


\section{eJRIEPS 23 avril 2011}

Enfin à propos du registre de participation, nous remarquons qu'un même entraîneur déploie une activité complexe avec parfois des préoccupations concurrentes, rejoignant en cela des travaux antérieurs (Saury, Durand, Theureau, 1997). II s'agit notamment :

- D'observer et d'analyser le jeu en se détachant de la dimension affective de l'évènement ;

- De centraliser un réseau d'informations et de communications avec différents interlocuteurs: l'autre entraîneur, le manager dans les tribunes, éventuellement le vidéo statisticien, le staff médical, l'arbitre, le délégué au bord du terrain et bien sûr les joueurs ;

- De gérer les remplacements des joueurs tout en restant concentré sur le jeu: remplissage des fiches, consignes au joueur entrant et commentaires à celui qui sort ;

- Éventuellement en tant qu'adjoint de terrain, de porter le tee au buteur ;

- Et dans un staff, d'exercer une fonction de tutorat avec un entraîneur moins expérimenté.

L'entraîneur est ainsi amené à tenir différents rôles durant le match. II est important que la répartition des rôles soit bien définie au sein du staff technique, en fonction des compétences de chacun et des registres préférentiels de maîtrise et de lecture de chaque entraîneur. Or nous avons pointé des styles de coaching avec un fonctionnement préférentiel en autonomie ou au contraire une collaboration en réseau.

2. 2. Activité des joueurs de rugby en match

Mouchet (2008a) étudie la subjectivité dans les décisions tactiques des joueurs de rugby d'élite en match. Nous proposons d'examiner aujourd'hui ces résultats relatifs à l'activité décisionnelle, à travers le filtre des registres de technicité, en tentant de souligner l'interaction dans la modélisation suivante. 


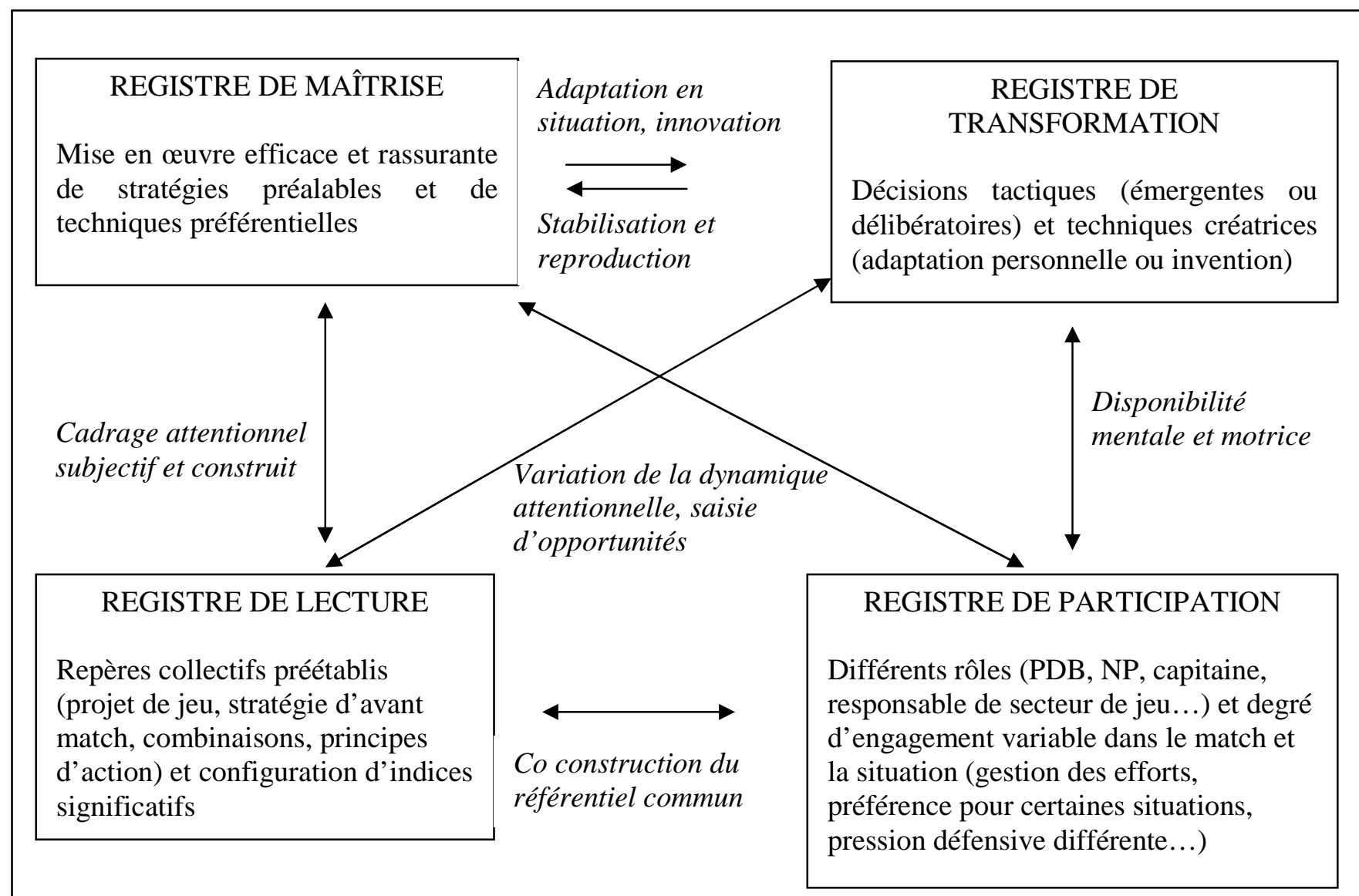

Figure 1 Les registres de technicité comme grille d'analyse de l'activité décisionnelle des joueurs

Cette figure tente de spécifier les différents registres de technicité pour étudier un objet particulier, l'activité décisionnelle du porteur de ballon en match. Cette modélisation nous semble respecter les critères d'identification des registres de technicité selon Glomeron (2001). En effet la technicité :

- S'exprime dans une action finalisée par un souci d'efficacité ;

- Fonctionne dans une situation de jeu inhérente au système sociotechnique du match ;

- Est généralisable à un ensemble de situations et d'acteurs, ici des joueurs impliqués dans les phases de mouvement général où les joueurs et le ballon sont en mouvement (Deleplace, 1979) ;

- Correspond à une activité cohérente et reproductible pour l'acteur, la décision étant ici le pôle organisateur de l'ensemble des registres.

Cette modélisation met en avant à travers les flèches à double sens et l'enveloppe générale qui enserre l'ensemble, les interactions entre les registres au sein d'un système. 


\section{eJRIEPS 23 avril 2011}

Cela permet de souligner le caractère dynamique, évolutif, fonctionnel de cette activité. Ainsi que l'articulation entre des éléments personnels et des aspects collectifs. Ou encore la combinaison de ressources de différente nature mobilisées dans l'activité technique du sujet, aspect mis en évidence par Bouthier (1993) avec les composantes de l'action sportive.

Nous pouvons penser qu'un registre dominant peut être organisateur de l'ensemble, avec des différences selon les joueurs. Ainsi nous avions remarqué que certains sujets étaient efficaces en appliquant précisément les décisions stratégiques, donc plutôt le registre de maîtrise. Mais les résultats faisaient aussi apparaître une compétence commune qui consiste à utiliser la diversité des modes de fonctionnement décisionnel, entre des décisions en acte émergentes, et des décisions où l'activité réflexive de type délibératoire prédomine. Cette flexibilité qui s'exprime selon les degrés de pression dans le rapport de force, est source d'adaptation tactique aux opportunités à saisir en cours d'action. Ce résultat peut être envisagé à travers le prisme des registres de technicité. En effet la flexibilité est au cœur de l'articulation entre :

- Le registre de maîtrise, dans ce cas les stratégies préalables, la programmation des techniques individuelles et collectives ;

- Le registre de transformation, ici l'adaptation tactico-technique favorable à la création et/ou l'exploitation d'un déséquilibre dans le rapport de force ;

- Le registre de lecture, plus exactement la dynamique attentionnelle avec la construction subjective d'une configuration d'indices significatifs, permettant la reconnaissance de repères préalables mais aussi l'identification d'opportunités à exploiter. Ou encore les changements de cadrage attentionnel permettant l'anticipation ;

- Le registre de participation, avec une disponibilité mentale et motrice favorable à l'adaptation dans un contexte local particulier.

\section{Conclusion}

Deforge (1993) définit la technologie comme une conquête de la réflexion sur un champ de pratiques et de savoir par les acteurs eux mêmes... Que la culture technique soit pensée, parole, éthique et acte du producteur autant que du consommateur de technique. Dans cette perspective et plus spécifiquement dans le champ des APSA, nous considérons en référence à Bouthier (2008), que l'un des enjeux des recherches technologiques consiste à travailler à partir des savoirs d'action des pratiquants, pour les caractériser, les comprendre, les modéliser, les capitaliser, afin de les optimiser en retour. 


\section{eJRIEPS 23 avril 2011}

Nous souhaitons alors mettre en avant quelques pistes de réflexion concernant la pertinence et le caractère fonctionnel de cet outil conceptuel (les registres de technicité), pour décrire et analyser l'activité des sujets dans la pratique d'APSA.

Tout d'abord, soulignons le fait que ces registres peuvent être exploités différemment à des fins de recherche ou de formation. Soit en se focalisant sur l'un d'entre eux, comme Uhlrich (2005) qui s'intéresse principalement au registre de lecture, ou comme nous avons illustré les compétences des entraîneurs dans les différents registres. Soit en mettant en lumière la dynamique d'articulation des registres, comme nous l'avons fait à propos de l'activité décisionnelle des joueurs dans une situation de jeu de mouvement. Nous estimons qu'il convient alors d'envisager un système avec un registre dominant et des registres présents de manière secondaire selon la situation étudiée. C'est donc le contexte de la situation qui donne du sens à une configuration particulière des registres pour un sujet donné. Cependant nous pouvons également envisager un registre préférentiel chez un même sujet, plus prégnant que les autres, et cela indépendamment du contexte.

D'autre part, notons que la dimension temporelle est présente dans l'articulation des registres. Dans l'instant présent et bref où un sujet met en œuvre des actions originales au regard d'une situation particulière. Mais aussi en ce qui concerne le long terme avec par exemple l'idée d'évolution, d'invention de techniques novatrices. Dans le deuxième cas nous pouvons citer en rugby l'exemple de l'utilisation de la passe au pied longue et transversale pour un partenaire démarqué, afin de lober le premier rideau défensif et d'exploiter au mieux la largeur du terrain. L'utilisation de cette technique s'est développée depuis environ 3 ans en rugby de haut niveau, alors qu'elle n'était que l'apanage de quelques joueurs. Elle est devenue une arme offensive utilisée plus fréquemment, avec une façon adaptée de tenir le ballon et de taper sous la pointe de ce dernier. Cette technique est entrée dans sa phase de stabilisation, c'est-à-dire que nous passons d'un registre de transformation vers le registre de maîtrise. Cette adaptation technique est identifiée, formalisée et transmise. Le problème est de préserver le sens de son utilisation dans le contexte d'opposition, sous peine de tomber dans une formation techniciste. La prise en compte de la dimension diachronique offre un regard complémentaire à une étude synchronique plus révélatrice de la configuration momentanée des registres.

Enfin ce concept conçu à l'origine dans le champ de la formation des enseignants des disciplines technologiques, nous paraît utile dans le champ de la formation des intervenants en STAPS. II contribue à offrir à la communauté des entraineurs un savoir technique, des instruments éprouvés, et des moyens originaux d'analyse de l'activité technique des sujets et des compétences en acte. Bouthier (2008) signale à ce propos 


\section{eJRIEPS 23 avril 2011}

que des recherches récentes en technologie des APSA «autorisent une remise en perspective des rapports réciproques entre pratiques professionnelles et activités de formation, notamment à travers l'étude des savoirs utiles pour l'action, des expériences favorables à leur reconstruction et des compétences techniques ainsi développées. » 5 Nous souhaitons également souligner la proximité de préoccupations et la complémentarité qui existent entre l'approche technologique en STAPS et la didactique professionnelle. En effet cette dernière a comme objectif de repérer les compétences mobilisées dans une activité, afin de construire des contenus de formation correspondant à la situation professionnelle de référence, en utilisant les situations de travail comme des supports pour la formation des compétences (Pastré, 2002). Reffugi, Louis, Attiklémé, Deridder (2006) ont ainsi conçu et mis en œuvre un dispositif de formation mettant les étudiants en situation de micro-enseignement (d'intervention sur leurs pairs) qui intègre le développement des compétences d'observation et d'interprétation des conduites motrices. Ils ont ensuite analysé comment le dispositif élaboré collectivement fut utilisé par les formateurs. L'étude montre une appropriation différenciée selon les formateurs dans les différentes APSA; elle témoigne également de la difficulté éprouvée par les étudiants dans la construction d'une compétence à l'observation et à l'analyse des conduites motrices de leurs pairs. Nous pouvons penser que la grille d'analyse des registres de technicité pour caractériser l'activité technique des sujets, peut être mobilisée dans ce type de formation.

Par conséquent le passage d'un concept outil pour caractériser et définir l'action des enseignants de technologie et d'EPS au regard des pratiques sociotechniques de référence et ce à des fins de formation, à un concept outil pour analyser l'activité des entraîneurs et des joueurs de rugby, nous semble constituer une piste intéressante. Ce changement de fonction ne nous paraît pas entraîner une perte de sens car les registres de technicité concernent toujours l'activité technique des sujets. Nous tentons en plus de mettre en avant la double facette publique et privée de cette technicité, et de préciser les relations dynamiques entre les registres. Enfin cet usage renouvelé trouve également un intérêt en vue de la formation. Cette dynamique conceptuelle est une caractéristique du savoir technologique selon Staudenmaier (1988) ou Bouthier (1993) pour qui les concepts scientifiques ont besoin d'être restructurés, redéfinis en fonction du problème et de la demande spécifique pour pouvoir contribuer aux connaissances technologiques. Les sciences sont ainsi exploitées selon une dynamique, une rationalité et une finalité propres

\footnotetext{
${ }^{5}$ Bouthier, D., Technologie des APSA : évolution des recherches et de leur place dans le cursus STAPS, p.50
} 


\section{eJRIEPS 23 avril 2011}

à la technologie, laquelle ne se réduit pas à une application de connaissances scientifiques.

\section{Bibliographie}

Amade-Escot, C., Bos, J.C., Dufor, F., Dugrand, M., Orphee, B. \& Terrisse, A. (1994). Didactique et culture technique. Spirales, 7, 8-18.

Bouthier, D., (1993). L'approche technologique en STAPS, représentations et actions en didactique des APS. Habilitation à diriger les recherches, Université Paris Sud, Orsay.

Bouthier, D. (1995). L'EPS et son rapport aux techniques. Spirales, 8, 96-98.

Bouthier, D. (2008). Technologie des APSA : évolution des recherches et de leur place dans le cursus STAPS. eJRIEPS, 15, 44-59.

Bouthier, D. \& Durey, A. (1994). Technologie des APS. Impulsions, 1, 95-124.

Combarnous, M. (1982). La culture face à la technique. Culture Technique, 7, 227-241.

Deforge, Y. (1993). De l'éducation scientifique à la culture technique; pour une maîtrise sociale de la technique. Paris : ESF, Collection Pédagogies.

Deleplace R. (1979). Rugby de mouvement, rugby total. Paris : Revue EPS.

Durey, A. (1995). L'EPS et son rapport à la technique. Spirales, 8, 93-95.

Eloi, S. (2009). Style d'un passeur de haut niveau en volley-ball. eJRIEPS, 17, 76-105.

Gérard, J-P. \& Bouthier, D. (2004). Apprendre à lire le mouvement : expérimentation d'un instrument de formation. eJRIEPS, 6, 23-39.

Glomeron, F. (2001). Unité et cohérence de la formation des professeurs de technologie au collège - Contribution à la définition des registres de technicité et des compétences professionnelles nécessaires. Thèse de doctorat en Sciences de l'Éducation, École Normale Supérieure de Cachan, Cachan.

Gréhaigne, J.F. \& Bouthier, D. (1994). Analyse des évolutions entre deux configurations du jeu en football, Science et Motricité, 24, 44-52.

Heidegger, M. (1954, Trad. André Préau 1958). Essais et conférences, Paris, Gallimard.

Leplat, J. \& Pailhous, J. (1981). L'acquisition des habiletés mentales : place des techniques. Le Travail Humain, 44, 2, 275-282.

Martinand, J.L. (1994). La didactique des sciences et de la technologie et la formation des enseignants. Aster, 19, 61-75.

Mouchet, A. (2008a). La subjectivité dans les décisions tactiques des joueurs experts en rugby. eJRIEPS, 14, 96-116. 


\section{eJRIEPS 23 avril 2011}

Mouchet, A. (2008b). Le coaching des sélections nationales de rugby en match. In P. Fleurance \& S. Pérez (Eds.). Interrogations sur le métier d'entraîneur(e). (pp. 281288). Les Cahiers de l'INSEP, 29, Paris, INSEP,.

Mouchet, A. (2010). Le coaching des sélections nationales en rugby: observation et analyse du jeu en début de match. In C. Amans-Passaga, N. Gal Petitifaux, P. Terral, M. Cizeron \& M.F. Carnus (Eds.). L'intervention en sport et ses contextes institutionnels : cultures et singularité de l'action. (pp. 81-86). Albi : Presses du Centre Universitaire Champollion.

Mouchet, A. \& Le Guellec, L. (2010). Les communications entraîneurs/joueurs en match de rugby international. Communication orale au 6e Colloque de l'Association pour la Recherche sur l'Intervention en Sport (ARIS), Sherbrooke, 27, 28 et 29 mai 2010.

Mouchet, A., Uhlrich, G. \& Bouthier, D. (2008). Dynamique de production du jeu de mouvement en rugby: tendances communes et Particularités. Sciences et motricité, 64, 93-102.

Mouchet, A., Amans-Passaga, C. \& Gréhaigne, J.F. (2010). L'approche technologique. lin M. Musard, M.P. Poggi \& G. Carlier (Eds.). Sciences de l'intervention en EPS et en sport : résultats de recherches et fondements théoriques. (pp. 201-222). Paris, EPS.

Pastré, P. (2002). L'analyse du travail en didactique professionnelle. Revue Française de Pédagogie, 138, 9-17.

Refuggi, R., Louis, E., Attiklémé, K. \& Deridder, M. (2006). Intervention et appropriation de « savoirs » sur les techniques sportives. Une innovation en formation universitaire des étudiants de licence STAPS. eJRIEPS, 9, 67-79.

Saury, J., Durand, M. \& Theureau, J. (1997). L'action d'un entraîneur expert en voile en situation de compétition : étude de cas. Contribution à une analyse ergonomique de l'entraînement. Sciences et Motricité, 31, 21-35.

Uhlrich, G. (2005). Rôle des artefacts matériels et cognitifs dans le développement des compétences de l'intervenant éducatif en rugby. Thèse de Doctorat (non publiée), Université Bordeaux 2, Bordeaux.

Uhlrich, G. \& Bouthier, D. (2008). Efficacité d'une situation de formation, par la simulation, à l'observation du mouvement général en rugby. eJRIEPS 15, 198-215.

Vermersch, P. (2003). Des origines de l'entretien d'explicitation aux questions transversales à tout recueil de verbalisation à posteriori. Expliciter, 50, 16-35. 